\title{
REVIEW
}

\section{Research progress on genetic improvement of Betula platyphylla Suk.}

\author{
Chuanping YANG (凶) \\ State Key Laboratory of Tree Genetics and Breeding, Northeast Forestry University, Harbin 150040, China
}

\begin{abstract}
Betula platyphylla Suk. is one of the most widely distributed species of Betula, the fourth most valuable timber species in north-eastern China and also a common tree species for landscaping. Over the past 30 years, effective progress has been made in genetic improvement and molecular breeding of B. platyphylla. There has been extensive research on breeding techniques, including the collection and conservation of B. platyphylla germplasm resources, provenance trials, intensive breeding techniques, crossbreeding and asexual propagation techniques, ploidy breeding and mutation breeding technology, genome sequencing, gene cloning, transgenic and molecular mechanisms of wood formation. A Betula germplasm resource collection has been established by collecting different provenances, and full-sib and half-sib families. In addition, the geographic variation patterns of $B$. platyphylla provenances have been revealed, and the provenance division and superior provenance selections made. B. platyphylla flowering and seeding have been improved through intensive breeding techniques. Interspecific hybridization, intraspecific hybridization and parallel crosses were made using fine parents, and $B$. platyphylla intensive seed orchards have been established. Systems of asexual propagation, including cuttings, grafting and tissue culture have been established. A $B$. platyphylla tetraploid was successfully constructed and a B. platyphylla triploid seed orchard established. The growth, wood property and resistance genes of $B$. platyphylla have been cloned. An efficient B. platyphylla transgenic system mediated by Agrobacterium tumefaciens was established, and genes encoding B. platyphylla insect resistance, drought resistance and salt tolerance, lignin synthesis, flowering, hormone transport and balance obtained. B. platyphylla molecular markers were developed and the high density B. platyphylla genetic map constructed. All this research
\end{abstract}

Received September 15, 2017; accepted November 20, 2017

Correspondence: yangcp@nefu.edu.cn has provided a model and data for the foundation of forest genetic improvement and applied research.

Keywords Betula platyphylla, genetic improvement, molecular breeding, seed orchard

\section{Introduction}

B. platyphylla Suk., also named Asian white birch, naturally occurring in East Asia, is a widely distributed species and the fourth most valuable timber species in north-eastern China and a common tree species for landscaping. Betula bark and juice have high medicinal value. Since the 1990s, B. platyphylla has been listed as one of the tree species in Chinese science and technology projects. B. platyphylla has been systematically researched by the core research teams of Northeast Forestry University. The study mainly covers standard breeding, ploidy and mutation breeding and molecular breeding. In this paper, we review the key research progress in genetic improvement of $B$. platyphylla.

\section{Standard breeding research on Betula platyphylla}

2.1 Construction of core germplasm and collection of germplasm resources of Betula platyphylla

Germplasm resources are important for seed selection and production. Therefore, the collection, preservation, research and utilization of forest seed resources are highly valued by domestic and foreign breeders. The increasing number of germplasm banks have brought significant challenges for their preservation, evaluation, research and utilization. Frankel put forward the concept of core germplasm on the basis of germplasm banks ${ }^{[1]}$. The aim was to minimize the number of germplasm resources to maximize the preservation of the genetic diversity of the entire population, so as to solve the difficulties and 
utilization problems of large-scale germplasm resources ${ }^{[2]}$. B. platyphylla primary core germplasm resources has been evaluated according to diameter at breast height (DBH), tree height, volume and cellulose concentration data, based on 240 B. platyphylla families, using different sampling rates $(5 \%, 10 \%$ and 15\%), two distance calculation methods (Mahalanobis distance and Euclidean distance), eight kinds of system clustering methods (the shortest distance method, furthest-neighbor method, intermediate distance method, gravity model method, average-linkage method, weighted pairing arithmetic mean method, changeable method and the sum of squared deviation method) and three sampling methods (random sampling method, priority sampling method and deviation sampling method), as well as the evaluation methods such as mean difference percentage, variance difference percentage, range coincidence rate and the variable rate of coefficient of variation. The results identified the best representative primary core germplasm of $B$. platyphylla in 24 families which were delineated using the $10 \%$ sampling ratio, Mahalanobis distance, the shortest distance system clustering method and the priority sampling method ${ }^{[3]}$.

Since the Ninth Five-year Chinese National Programs for Science and Technology Development, the collection of Betula germplasm resources has been ongoing, including European Betula pendula, Kyrgyzstan $B$. pendula, B. pendula $c v$. Purple Rain, B. platyphylla f. laciniata, B. platyphylla var. japonica and accessions from Finland, Canada, Russia, Kazakhstan, Japan and other countries. It also includes more than 30 provenances of B. platyphylla from different Chinese provinces, and genetic material from more than 210 full-sib and half-sib families. Thirteen B. platyphylla germplasm resources have been established in preservation and demonstration forests of over 40 ha in north and north-east China, and other regions, which provides a wealth of resources for $B$. platyphylla seed selection and production.

\subsection{Provenance trial research on Betula platyphylla}

Provenance evaluation is one of the main methods of tree selection. Through the provenance evaluation, we can study the relationship between the geographical variation, size variation and the tree species, and ecological environment and evolutionary factors. On this basis, the suitable provenances can be chosen for afforestation and seed zone planning programs, and rules established for certain geographical areas. Since the 1990s, B. platyphylla provenance evaluation has been conducted three times, the first for the whole distribution area, and the latter two for north-east region sources. In addition, taking the other typical local provenance experiments into account, material properties including the growth traits, morphological traits, seed traits and planting material have been researched and reported ${ }^{[4-8]}$.
The geographical variation of $B$. platyphylla provenances have been determined. There were significant differences in traits between different provenances of B. platyphylla species that showed typical geographical variation patterns. The variation trends of growth traits, texture and wood property were bidirectional in longitude and latitude, with longitude taking priority. In contrast, the morphological and seed traits were dominated by latitude. The continuous distribution of provenances in the northern regions showed gradual variation, but the island-like distributed southern provenances showed ecotypic variation.

Provenance divisions of $B$. platyphylla were delineated. According to the results of the three provenance trials, 17 provenances were divided into five provenance areas: I Daxing'anling provenance area (including the Chauer and Moer Daoga sources); II Xiaoxing'anling, Zhang Guangcailing and Wandashan provenance area (including Wuyiling, Liangshui, Maoershan, Xiaobeihu and Dongfanghong provenances); III Changbaishan, Qianshan seed source area (including Qingyuan, Huanren, Caohekou, Huinan, Lushuihe and Wangqing provenances); IV Liupanshan and Qilianshan provenance area, (including Ningxia, Qinghai and Gansu provenances); V Tianshan provenance area (including Tianshan western provenance).

The superior provenances from each provenance area were selected, including fast-growing and high-yielding provenances: the superior provenances in Daxinganling area were Dailing and Wuyiling with genetic gain in height and volume of up to $35 \%$ and $24 \%$, respectively. The superior provenances in Xiaoxinganling, Zhangguangcailing and Wandashan areas were Wuyiling and Dongfanghong with genetic gain in height and volume of $42 \%$ and $27 \%$, respectively. The superior provenances in Changbaishan and Qianshan area were Bajiazi and Qinglong with genetic gain in height and volume of $32 \%$ and $18 \%$, respectively. Superior provenances for wood-based panel were Dailing and Heihe provenances in Daxinganling area with genetic gain in DBH (diameter at breast height) and volume of up to $26 \%$ and $22 \%$, respectively, Dongfanghong and Guandishan provenances in Xiaoxinganling, Zhangguangcailing and Wandashan area with genetic gain in DBH and volume of $31 \%$ and $25 \%$, and Bajiazi and Qinglong in Changbaishan and Qianshan area with genetic gain in DBH and volume of $36 \%$ and $21 \%$, respectively. In 2010, tree height, DBH, volume, carbon sinks and other indexes of 14-year provenances in three B. platyphylla provenance areas in Heilongjiang Province, Liaoning Province and Inner Mongolia Autonomous Region were analyzed, and four high-carbon sequestration provenances were selected, including Qinghekou, Qingyuan, Maoeshan and Xiaobeihu provenances in Heilongiang. The aboveground carbon storage of these four provenances in Heilongjiang increased by $47.2 \%, 22.4 \%, 20.8 \%$ and $17.3 \%$, respectively, when compared to the control. 


\subsection{Intensive breeding techniques for Betula platyphylla}

The intensive breeding technique is a method of greatly reducing the flowering and fruiting age of B. platyphylla in a plastic greenhouse by manipulating the action of temperature, light, $\mathrm{CO}_{2}$, hormones, fertilization and other interventions. After years of research, breakthroughs in intensive breeding of $B$. platyphylla have been achieved. The ecological factors display and control system was designed and established and has overcome the limitations of a variety of ecological factors which are difficult to control simultaneously in forest seed orchards and automates the collection of various ecological factors in the intensive breeding orchard. This provides first-hand information for revealing the mechanism of early flowering and robustness in B. platyphylla, and lays a theoretical foundation for intensive management and industrial production of other tree seeds. Six techniques to strengthen breeding were proposed, (1) application of $\mathrm{CO}_{2}$ to accelerate the transformation of vegetative growth to reproductive growth, (2) appropriate light intensity to promote flowering, (3) addition of flowering hormone and (4) girdling treatment to promote the differentiation of flower buds, (5) continuous light to promote differentiation of flower buds, and (6) flowering, temperature and humidity control to promote flowering. Therefore, B. platyphylla, which will flower and seed after 18 to 20 years under natural conditions, has been made to flower in 2 to 3 years, and seed in 3 to 4 years. On this basis, China's first $B$. platyphylla seed nursery was established. The breakthrough of $B$. platyphylla breeding techniques provides a model for intensive breeding of other species ${ }^{[9]}$. Also, second-generation seed nurseries of $B$. platyphylla have been established. The results of the progeny afforestation testing show that the improved seeds from the seed nursery had advantages of fast growth and good dryness compared to the control. At present, the intensive breeding technique has been extended to some areas in Heilongjiang, Jilin, Liaoning, Beijing and Inner Mongolia and other provinces and cities. At the same time, studies on reproductive biology and physiology of $B$. platyphylla were undertaken to elucidate the process of sexual reproduction in B. platyphylla ${ }^{[10-13]}$.

\subsection{Hybrid breeding techniques for Betula platyphylla}

Hybrid breeding is an important method to obtain new genotypes and create genetically modified forest materials. Through crossbreeding, interspecific hybridization and intraspecific hybridization have been achieved. Genetic parameters, such as general and special combining ability, were determined though diallel crossing ${ }^{[14-19]}$.

More than 80 interspecific hybrid combinations were obtained and three superior families were selected with height and DBH increased by $40 \%$ and $33 \%$, respectively, compared to the control. More than 150 intraspecific hybrid combinations were obtained and three superior families were selected with their height and DBH increased by $56 \%$ and $42 \%$, respectively, compared to the control.

Complete diallel hybridization can be used for controlled crosses to explore the general combining ability, special combining ability and reciprocal cross effects of the parents, providing a basis for parent selection for secondgeneration seed nurseries. The diallel hybridization was done with the Xiaobeihu improved provenance and the Maoershan improved provenance of B. platyphylla as the parents. The results showed that there were significant differences in the seedling traits of 25 hybrid combinations. The 1000-seed weight increased, in addition to the general and special combining abilities, and reciprocal cross effect of the germination rate and germination potential. The general combining ability of seedling heights was also notably different, however there was no significant effect on ground diameter. The difference between the special combining ability and reciprocal cross effect of seedling height and ground diameter were obvious and allowed the selection of hybrid combinations with increased height and DBH. Five good quality hybrid combinations were selected from the 25 hybrids and two superior parents identified ${ }^{[14]}$. There were significant differences in tree height, $\mathrm{DBH}$ and volume between the crosses of B. platyphylla and European B. pendula. The height, DBH and trunk straightness of eight-year-old hybrids were determined and analyzed by variance analysis, general combining ability, special combining ability and reciprocal cross effects. Based on these analyses, three superior parents and three hybrid combinations were selected with significantly improved tree height, DBH and straightness ${ }^{[16]}$.

2.5 Study of Betula platyphylla asexual propagation techniques

Asexual propagation is not only an important method for fixing favorable genotypes and obtaining improved populations and breeding of the improved cultivars, but also forms the basis for tree genetic engineering research. Therefore, propagation of B. platyphylla by cutting, grafting and tissue culture techniques have been studied.

The formation of adventitious roots in cuttings of B. platyphylla is by bark rooting, where the adventitious roots originate from the vascular ray cells at the intersection of the vascular cambium. There were no adventitious roots in the callus, revealing that the occurrence of callus had a negative effect on the formation of adventitious roots ${ }^{[20]}$. The IAA, ABA and soluble sugar concentrations in the shoots of $B$. platyphylla changed significantly, and the ratio of IAA/ABA was used as an index to measure the rooting ability of $B$. platyphylla. Rooting promoters significantly increased IAA and cytokinin (ZR) concentrations and reduced ABA and gibberellin (GA4) concentrations during root primordium 
initiation. The IAA/ABA and soluble sugar concentrations increased with the age of the parent tree. The rooting ability of different aged parent trees was not closely related to total nitrogen, but mainly determined by soluble sugar concentration. There was a significant difference in IAA/ ABA between clones. The rooting rate, as well as IAA/ $\mathrm{ABA}$ ratio, soluble sugar and $\mathrm{C} / \mathrm{N}$ was higher when only one leaf was retained on cutting slips ${ }^{[21]}$. In the process of shoot cutting, rooting accelerator and shooting state of the branch had a significant effect on rooting rate. There was a significant difference between the survival rate of cuttings at different transplanting times. The rooting rate was the highest when the one-year-old, semi-lignified softwood sprouts of root were used in cutting propagation, with the average rooting rate of above $70 \%$ and transplant survival rate of $62.5 \%{ }^{[22]}$. The shoot types, rooting media, clones and rooting promoters all had a significant effect on the rooting rate. The survival rate of cuttings was affected by factors such as transplanting time, number of main roots, cuttings and new leaf growth ${ }^{[23]}$.

Taking wild-type seedlings of $B$. platyphylla (5 to 6 years old) as rootstock, scions were collected from the 15to 17-year-old B. platyphylla trees and grafting done by ten different methods. These included water culture approach grafting (in the process of grafting, the lower part of the scions is kept in a bottle and cut off after grafting), medullary cambium grafting, and apical bud cleft grafting. The survival rate, growth rate and phenological characters of various grafting methods were systematically analyzed by analysis of variance, correlation analysis and partial correlation analysis. The results showed that the survival rate with the different grafting methods was significantly different, with water culture approach grafting and medullary cambium grafting having the highest success rates, $85.2 \%$ and $47.4 \%$, respectively. There were significant differences in the growth traits of different grafting methods. The growth and the leaf area were the highest with water culture approach grafting, followed by cleft grafting and cambium approach grafting. The germination, leaf stage and other phenological characters by different grafting methods were also quite different; for example, water culture grafting gave the earliest leaf stage and the leaf expansion rate can be used as an early predictor of graft survival rate ${ }^{[24]}$. Cuttings of $B$. platyphylla cv. Purple Leaf are best taken in August. Slips from the branch of one-year-old B. platyphylla cv. Purple Leaf were dipped briefly in $1 \mathrm{~g} \cdot \mathrm{L}^{-1}$ auxin and then put in a mix of peat soil, vermiculite and perlite $(5: 2: 3$ by volume) which allowed relatively easy rooting. Grafting propagation is best done in May with a one-year-old $B$. platyphylla as rootstock with improved grafting achieved by the wedge grafting and pith cambium grafting method on the stem about $5 \mathrm{~cm}$ above the ground. When two-year-old B. platyphylla was used as the rootstock, the wedge grafting method and the budding method worked best on a stem $45 \mathrm{~cm}$ above the ground. Grafting done on the lateral branches at 65 and $45 \mathrm{~cm}$ above the ground by layering and budding methods can give a higher survival rate $^{[25]}$.

In tissue culture, the optimized medium for different developmental stages was assessed. The best tissue culture system for B. platyphylla had a callus induction rate of over $70 \%$ and the differentiation rate of leaf and stem segments of over $90 \%$. This method can produce about 3000 adventitious buds after three generations of continuous cultivation of $B$. platyphylla clones with high differentiation rate. In the rooting stage, rooting rate was over $95 \%$ with an average number of two to five roots without using hormones. Concurrently, the bottleneck problem of B. platyphylla transplant survival rate was solved with a 1:1 peat:sand substrate giving a spring transplant survival rate of over $85 \%$. Overall, a total of 38 B. platyphylla tissue culture clones have been planted and more than 10000 seedlings bred ${ }^{[26-28]}$.

\section{Ploidy breeding and mutation breeding research on Betula platyphylla}

\subsection{Study of tetraploid induction of Betula platyphylla and establishment of a triploid seed orchard}

Ploidy breeding is an important approach for tree breeding by changing the number of chromosomes, producing different mutated individuals, and then selecting superior breeding methods for cultivating new cultivars. B. platyphylla tetraploids were induced with colchicine at a rate of $4 \%{ }^{[29]}$. The photosynthesis rate of tetraploids, as well as the net photosynthetic rate, PSII actual photochemical efficiency and photochemical quenching were significantly higher than those of diploids. The tetraploids were significantly different from diploids in terms of physiologic and hormonal level and the concentrations of soluble sugars, soluble protein, chlorophyll, auxin, abscisic acid and gibberellin of tetraploids were significantly higher than those of diploids. There were also increased numbers of inflorescenses in B. platyphylla tetraploids. Lengths of male bracts in tetraploids were greater than in diploids, as were infructescence length and diameter of tetraploids, as well as the pollen diameter. Changes in B. platyphylla tetraploid fertility were mainly seen in the male gametophyte. The pollen germination rate of $B$. platyphylla tetraploids was lower than for diploids. Transcriptome analysis showed that IAA and ethylene signal transduction pathways changed after genome doubling, which may be the cause of phenotypic changes ${ }^{[30]}$. The IAA and ABA concentrations in leaf buds of diploid, triploid and tetraploid $B$. platyphylla were determined by HPLC. The comparison of IAA and ABA concentrations in $B$. platyphylla leaf buds of different ploidy revealed that IAA concentration in polyploid B. platyphylla was higher, while the ABA concentration was significantly lower than 
in diploids. These results showed that the doubled genome had an important effect on endogenous hormone levels, which in turn affected the tetraploid phenotype ${ }^{[31]}$. At the same time, the maximum net photosynthetic rate of tetraploids was significantly higher than in diploids $(P<0.01)$. The difference between light saturation point, light compensation point and carboxylation efficiency was also significant $(P<0.01)$. The quantum yield of PSII in tetraploid chlorophyll fluorescence was significantly higher than in diploids $(P<0.05)$. Other parameters were not significantly different between the two groups $(P>0.05)$. These results indicate that these tetraploid $B$. platyphylla had stronger photosynthesis than the diploids $^{[32]}$. Moreover, the photosynthetic characteristics of tetraploid B. platyphylla seedlings were significantly improved under $\mathrm{NaHCO}_{3}$ stress ${ }^{[33]}$. The doubling of chromosomes also improved the wood quality of B. platyphylla, which had a significant effect on the fiber properties of wood; therefore superior parent trees for the pulp materials could be selected from the tetraploids ${ }^{[19]}$. The betulinic acid and betulin concentrations in the bark of $B$. platyphylla tetraploids were significantly greater, with the concentrations of three triterpenoids in tetraploid B. platyphylla generally higher than in diploids ${ }^{[34]}$.

After obtaining more than 400 B. platyphylla tetraploids, the triploid plants were obtained by hybridization between tetraploids and diploids. A total of 40000 triploid plants were obtained by hybridization and regional tests were conducted at ten sites. According to the results of regional tests a total of $31 \mathrm{~B}$. platyphylla tetraploids and 17 diploids were selected for phenotypic traits, seed traits and flowering, and a triploid seed nursery was established. Observations on the autotetraploid and heterotetraploid B. platyphylla parent trees and progeny in the seed nursery showed that there was a significant difference between the tetraploid and the diploid parent tree family, compared to the triploid family. The heterotetraploid parent trees and triploid offspring were superior to autotetraploid parent trees and offspring ${ }^{[32,35,36]}$.

\subsection{Space mutation breeding of Betula platyphylla}

Space mutation breeding refers to the process of using recoverable satellites to take plant seeds to high altitude, making them undergo genetic change in conditions such as microgravity, high vacuum, strong radiation and alternating magnetic field, from which new lines of these plants are selected on Earth. In recent years, space mutation breeding has become an important aspects of life science research in space. B. platyphylla seeds were carried by a recoverable satellite to subject them to space mutation. By analyzing the seed vigor and the characteristics of oneyear-old $B$. platyphylla seedlings of the four families after space flight, the results showed the following. The effect of space mutation on seedling height was significant and extreme; for example, a dwarfing phenomenon was observed after treatment. The effects on seed vigor, leaf chlorophyll concentration, net photosynthetic rate, MDA concentration and relative electrical conductivity were not significant. The activities of superoxide dismutase in four families were lower than those in the untreated control, and other variables differed within the families. Overall after space mutation, there was a beneficial effect on $B$. platyphylla seed vigor, the leaf chlorophyll concentration of seedling leaves decreased, the net photosynthetic rate increased slightly, and the MDA concentration and relative conductivity of leaves of three families were lower than those of the control ${ }^{[37]}$. The difference between tree height at two years old for the four treated B. platyphylla families and the controls was highly significant. The tree height for one family was significantly lower than the control, while for the other three families it was higher. The height difference between five-year-old B. platyphylla trees of two families and the control was significant. A total of 64 superior lines were selected with the average height increase of $14.8 \%$ compared to the control, with average DBH increasing by $27.9 \%$ and average volume by $64.3 \%$. These superior mutant strains lay the foundation for further development of B. platyphylla through progeny selection ${ }^{[38]}$.

\section{Molecular breeding techniques for Betula platyphylla}

\subsection{Gene cloning}

With the development of modern genomics technology, the genetic identification and cloning work has been rapidly increasing. Ten years ago, the number of full-length cDNAs cloned from $B$. platyphylla by constructing a suppression subtractive library combined with RACE technique was in single digits. Once second-generation sequencing technology was introduced, the number of full length $B$. platyphylla $\mathrm{cDNAs}$ was increased to hundreds by transcriptome sequencing. At present, 30626 genes of $B$. platyphylla have been identified by genome-wide sequencing. In addition, they can be analyzed by bioinformatics and gene expression profiles to screen for genes associated with specific biological processes. In the past ten years, the $B$. platyphylla genes related to flowering regulation, wood formation, and resistance to abiotic stress have been identified by homologous cloning, cDNA library construction, SSH library construction, or transcriptome sequencing and their expression pattern have been documented ${ }^{[30,39-47]}$.

\subsection{Transgenic Betula platyphylla}

Transgenic technology is an important means of investigating the function of genes and molecular breeding of B. platyphylla. An Agrobacterium-mediated B. platyphylla transgenic system has been established. The coculture 
medium was WPM (Woody Plant Medium) + BA $0.8 \mathrm{mg} \cdot \mathrm{L}^{-1}+$ NAA $0.02 \mathrm{mg} \cdot \mathrm{L}^{-1}+\mathrm{GA} 30.5 \mathrm{mg} \cdot \mathrm{L}^{-1}$. The antibiotic used for selection was $50 \mathrm{mg} \cdot \mathrm{L}^{-1}$ kanamycin. The optimal pre-culture time for leaf blade inoculation was $6 \mathrm{~d}$ with a new wound cut before inoculation. Coculture for $2 \mathrm{~d}$ inhibits Agrobacterium growth, prevents rot, and resulted in high transformation efficiency. Coculture and Agrobacterium elimination using a liquid system was better than a solid system. The best explants were stem segments with terminal buds and axillary buds and large leaves. Resistant calli were produced two months after inoculation with a transformation rate of $22 \%{ }^{[48]}$. Transgenic silencing, gene expression stability and expression levels after continuous subculture were some of the factors affecting transgenic breeding. The expression of exogenous genes in transgenic $B$. platyphylla was studied in subcultures after 4, 7, 9 and $15 \mathrm{~d}$ by quantitative PCR. The results indicated that the expression of the exogenous genes decreased with the increase in time of subculture, which was related to the methylation of DNA. The expression of the exogenous gene could be reactivated by 5 -azacytidine treatment ${ }^{[49]}$. Due to the long transgenic cycle of $B$. platyphylla, the study on the regulation of exogenous genes expression has been restricted, nevertheless, Agrobacterium-mediated B. platyphylla transient transformation technology can achieve the expression of exogenous genes economically and efficiently. Experiments revealed that the exogenous gene could be overexpressed or inhibited transiently in B. platyphylla using an Agrobacterium-mediated transient transformation system $^{[50]}$.

Many studies of transgenic B. platyphylla have been conducted and progress has been made in insect resistance, drought and salt tolerance, and regulation of flowering, growth and wood formation, depending on the genes and transformation system used.

Metabolites of B. platyphylla are abundant, and are the feeding substrate of many insects. The damage caused by insect pests has resulted in a great economic loss in B. platyphylla forests. Chemical methods to control pests have shortcomings, such as heavy workload, environmental pollution and development of insecticide-resistant pests. Transgenic insect-resistance provides a new way to control pests in B. platyphylla forests. The chimeric genes of spider insecticidal peptide and the $C$ peptide sequence from the $B t$ gene were introduced into the $B$. platyphylla genome and ten transgenic $B$. platyphylla insect-resistant lines were obtained. The results showed that the transgenic B. platyphylla could inhibit the growth of gypsy moth and delay its development, with a death rate of over $75 \%{ }^{[48]}$. Further experiments showed that transgenic $B t$ gene in B. platyphylla caused serious damage to the mid-gut of the infected gypsy moth and consistently reduced the development rate and body mass of larvae ${ }^{[51]}$. Currently, insectresistant transgenic B. platyphylla, which has been granted a pilot license for transgenic forest trees by the State
Forestry administration, is undergoing regional evaluation.

B. platyphylla provides excellent pulp material, and the new $B$. platyphylla transgenic cultivars with modified lignin concentration are important for the breeding of pulp wood. Key enzymes of lignin biosynthesis have been identified in B. platyphylla, and studies of lignin-synthesisrelated genes conducted. Thirty nine BpCCR1 (Cinnamoyl-CoA reductase in birch) inhibited expression lines and 19 BpCCR 1 overexpressed lines were obtained. Lignin concentration and cell wall thickness increased in overexpressed lines, and decreased in inhibited lines ${ }^{[52]}$. Three overexpressed $B p C C R 2$ gene lines were obtained, and the lignin concentration in transgenic lines increased to different degrees, which demonstrated the role of $B p C C R 2$ gene in the regulation of lignin biosynthesis. The lignin composition of transgenic BplCCoAOMT $B$. platyphylla was altered, as this gene is involved in the synthesis of S-type monomer lignin. Eleven B. platyphylla lines with antisense COMT1 gene had biosynthesis of lignin inhibited. This research on lignin biosynthesis genes in transgenic B. platyphylla has laid the foundation for the genetic improvement of wood properties.

Flower development is an important process in sexual reproduction of plants. The formation of floral meristems can be controlled by transgenes such as meristem specific genes and flowering time gene, so as to affect the flowering time of plants, to give early or delayed flowering. The $B p A P 1$ gene was transferred into B. platyphylla, and 17 transgenic lines were obtained (five were overexpressed and 12 inhibited). These transgenic plants flowered in the tissue culture bottles after two months. BPAPl genetic engineering breeding greatly shortened the flowering cycle of $B$. platyphylla and provided experimental material suitable for the study of important genetic traits in B. platyphylla ${ }^{[53]}$.

Afforestation in difficult sites is one of the important aspects solved by genetic improvement of trees and using transgenics to study drought resistance and tolerance to salt and alkali is of great value for the genetic improvement of $B$. platyphylla. Transfer of an ethylene response factor gene BpERF11 into B. platyphylla showed that the gene negatively regulates salt tolerance and osmotic stress, so inhibition of expression of this gene can improve the stress resistance of $B$. platyphylla ${ }^{[47]}$. By transferring another MYB transcription factor gene into $B$. platyphylla, the abiotic stress tolerance of overexpressed lines was significantly improved, indicating the positive effect of this gene on stress resistance ${ }^{[54]}$. The heterogenous Tamarix TaLEA-ThbZIP gene was transferred into $B$. platyphylla and nine transgenic lines showing drought resistance and salt tolerance were obtained, including five transgenic lines containing $L E A$ genes which could grow normally in soil containing $0.5 \% \mathrm{NaCl}$ and four transgenic lines expressing bZIP genes. The results indicated that these transgenic lines had improved drought resistance and salt tolerance ${ }^{[55]}$. 
In addition, the transcription factor BpMYB106 could significantly increase the density of trichomes and the growth rate of transgenic $B$. platyphylla ${ }^{[56]}$. Expression of BpGH3.5 in transgenic B. platyphylla decreased the length and the number of cells in the apical meristematic zone of the root, thus the root length and root biomass were decreased in transgenic plant compared to the control.

\section{Molecular mechanism of wood formation in Betula platyphylla}

\subsection{Construction of cDNA library of Betula platyphylla and seasonal expression analysis of wood related genes}

A cDNA library is an effective resource for studying regulation of gene expression, including genes expressed in specific tissue. In the cDNA library of the B. platyphylla cambium, 154 ESTs that may be associated with cambium development and wood formation were identified, representing 50 unigenes. Among these were 17 full-length cDNA sequences related to lignin synthesis, cell structure, cell wall polysaccharide synthesis and developmental regulation. The expression patterns of 14 genes related to wood formation in B. platyphylla were studied at different times during a growing season. The results showed that in the wood formation process in B. platyphylla, the expression levels of these genes significantly increased during the two periods, 26 April to 11 June and 30 July to 18 September, suggesting that these may be the important periods for wood formation in B. platyphylla. The genes relating to cell wall formation, cell expansion, and the synthesis of cellulose and lignin revealed a high expression level from 26 April to 11 June, indicating that this period might be the important stage of cell wall formation and lignin biosynthesis in B. platyphylla. After 30 July, expression of genes related to cell wall rigidity, such as $P O D, C C R$ and $X E T$, increased, suggesting that during this time, xylem cells gradually stopped growing and cell wall rigidity increased ${ }^{[57]}$.

\subsection{Transcriptome and proteome analysis of Betula platy- phylla tension wood}

Tension wood is a useful location to study the gene expression and identification of genes for wood formation. To investigate the regulation of gene expression during tension wood development, three transcriptomes from tension, opposite and normal wood (TW, OW and NW, respectively) were established by artificially simulating the effect of gravity on the stem of B. platyphylla. A total of 80909 unigenes were obtained using de novo sequencing. A series of high abundance genes, such as those encoding lipid transfer protein and xyloglucan endo-transglycosylase/hydrolase were obtained. Analysis of gene expression differences among the three transcriptomes showed that there were 6264 differentially expressed genes between TW and NW, 4533 differentially expressed genes between OW and NW, and 4025 differentially expressed genes between OW and TW. Genes encoding key enzymes of lignin biosynthesis were identified, including PAL, COMT, $C 3 H, 4 C L, C C R$ and $C A D$. GO enrichment analysis showed that these differentially expressed genes were closely related to the molecular functions and biological processes involved in wood formation. In the process of TW formation, cellulose-synthesis-related genes were induced and lignin-synthesis-related genes inhibited. Transcription factor genes showed different expression patterns, suggesting the complexity of gene regulation involved ${ }^{[31]}$.

Proteins are the direct performers of biological functions. In a study of the regulation of protein concentrations during the process of TW formation, a four-year-old $B$. platyphylla was subjected to artificially simulated gravity. The developing xylem of TW, OW and NW was sampled after 4 weeks of treatment and their proteomics compared using the iTRAQ technique. A total of 1104 distinct differentially-expressed proteins were identified. Pathways that were significantly enriched in these proteins were identified by super geometry inspection. The results showed that most of the proteins associated with lignin synthesis were downregulated in TW compared to OW or NW, including seven key enzymes that functioned in phenylpropanoid biosynthesis and lignin monomer biosynthesis, indicating the synthesis of lignin in TW is inhibited. Most of the proteins associated with polysaccharide metabolism were upregulated in TW, indicating that the biosynthesis of polysaccharides was promoted in this process. In particular, accumulation of sucrose, fructose and $\alpha$-D-glucose could contribute to the synthesis of cellulose. The associated enzyme proteins in the TCA cycle were upregulated in TW compared to OW or NW. The results showed that energy transport and modification of cell walls are promoted during TW development.

\subsection{Expression profiling of primary and secondary xylem in Betula platyphylla}

Identification of gene expression profiles in stem segments at different developmental stages can help in the study of molecular regulation of xylem during primary and secondary growth. In the gene expression profiling of first, third and fifth internodes of B. platyphylla, differentially expressed genes at different developmental stages were identified. Combed with anatomical analysis, the results showed that differential expression genes were enriched between the first and third, as well as first and fifth internodes, where xylem development stages were obviously different. In contrast, there were no significant differences in gene expression between the third and fifth internodes. In the primary tissues, the expression of genes related to cell wall expansion and primary cell wall 
cellulose synthesis was higher than in secondary tissues. The expression of lignin synthesis, secondary wall cellulose synthesis, cell wall modification and xylem extension-related genes in secondary stems were significantly higher than those in primary stems. Plant hormoneresponse genes and plant transcription factors had complex patterns of expression. Indeed, different family members participated in different stages of development ${ }^{[58]}$.

\subsection{Regulation of xylem development by gibberellin in Betula platyphylla}

Gibberellin (GA) is a key signaling factor for vascular differentiation, fiber development and lignification. Studies on the changes in xylem development and gene expression under GA treatment can help in understanding the regulatory mechanism of hormone signaling in wood formation. The seeds and seedlings of $B$. platyphylla were treated with GA3 and/or paclobutrazol (PAC) and the germination rate, xylem development and secondary cell wall development measured. The changes in xylem-related gene expression were analyzed. The results showed that GA could improve seed germination rate, germination potential, germination index, hypocotyl length and fresh weight of seedlings, but these indices were inhibited by PAC. Sections of hypocotyls and seedling stems stained with toluidine blue or phloroglucinol- $\mathrm{HCl}$ showed that the xylem development was promoted by GA, resulting in an increase in the width of the xylem. The expression of $N A C$ gene and $M Y B$ transcription factors, CESA, PAL and $G A$ oxidase were analyzed by qRT-PCR. The results revealed that the expression of these genes was induced under GA treatment, suggesting that they are involved in regulation of xylem development induced by GA. The study showed that GA could promote the development of xylem and therefore induce the expression of related genes in B. platyphylla ${ }^{[59]}$.

\section{Development of molecular markers and construction of genetic linkage maps in Betula platyphylla}

Using the closely linked characteristics of molecular markers and the target trait genes, the presence of the target gene can be identified by detecting the molecular marker and used as a means of selecting the target trait in a breeding program. It has the advantage of being fast, accurate and unaffected by environmental conditions, which can be used as a means to identify the relationship between parents, the transfer of quantitative traits and recessive traits in backcross breeding, the selection of hybrid progeny, the prediction of heterosis and the identification of purity of a line. For molecular markers in B. platyphylla, RAPD ${ }^{[60]}$, ISSR-PCR ${ }^{[9]}, \mathrm{SSR} \mathrm{PCR}^{[61]}$, $\operatorname{AFLP}^{[6]}$ and SRAP ${ }^{[62,63]}$ systems were established and optimized. The number of SRAP primers did not affect the change of genetic parameters between different provenances of $B$. platyphylla. Increasing the number of primers, however, improved the ability to distinguish genetic differences between different provenances. With 15 pairs of SRAP primers, the percentage of detectable polymorphic loci was above $98.5 \%{ }^{[55]}$. Of $583 \mathrm{~B}$. platyphylla trees, 100 were selected after measuring the length of individual fibers. Then genomic differences were analyzed by the random amplified polymorphic DNA (RAPD) technique. The molecular markers significantly correlated with fiber length when screened by multiple regression analysis between amplified bands and traits. After screening with 20 RAPD primers, seven fragments of six primers were significantly correlated with fiber length. The correlation coefficient between segment BFL and fiber length was $0.401(P<0.05)$. This fragment was cloned and sequenced and then successfully transformed into a sequence characterization amplified region (SCAR) marker associated with long fiber traits. The marker was $80 \%$ effective for long fibers in B. platyphylla ${ }^{[64]}$. The genomic differences were analyzed using inter-simple sequence repeats (ISSR), and these significantly correlated with the fiber length in B. platyphylla. After screening with 20 ISSR primers, four fragments of four primers were significantly correlated with fiber length, with the regression coefficient of fragment "BFLI-3" and fiber length of -0.331 $(P<0.01)$. This fragment was cloned and sequenced and successfully transformed into SCAR markers associated with long fiber traits. The marker was $76 \%$ efficient for short fibers B. platyphylla ${ }^{[65]}$.

Construction of genetic maps for B. platyphylla has undergone a process of gradual refinement. Based on the genetic map constructed using AFLP technology ${ }^{[60,66-68]}$, combined with genome sequencing, a high density B. platyphylla genetic map containing 7900 loci has been constructed by SLAF-seq (specific-locus amplified fragment sequencing) using a hybridized population of 200 trees, with B. platyphylla and Betula pendula as cross parents. The total map distance was $1270.8 \mathrm{cM}$ with the average genetic distance of $0.16 \mathrm{cM}$. The average mark integrity is $99.5 \%$. The mean depth of the parent is 60 times and the average depth of the offspring is 9.38 times.

\section{Future research}

(1) Based on the characteristics of B. platyphylla tree species, an effective breeding strategy and a model breeding program could be developed to make genetic improvement of $B$. platyphylla comprehensive, systematic and standardized, to strengthen the management of breeding groups and reproduction groups, and to maximize the genetic gain of superior lines.

(2) The collection, research, preservation and utilization of $B$. platyphylla gene resources could be strengthened. 
The acquired germplasm resources could be identified in-depth and evaluated to make full use of the existing germplasm resources. New genetic resources could be constantly added to broaden the genetic basis. B. platyphylla breeding populations with an enriched genetic basis could be built to develop and use these genetic resources.

(3) Early selection and shortening of breeding generations could be studied. To improve the breeding efficiency of B. platyphylla, there is an urgent need for the study of early selection and shortening of the breeding cycle. The early and late correlation of selected traits might be determined under the premise of intensive breeding, which effectively shortens the $B$. platyphylla improvement cycle to improve breeding efficiency.

(4) Further intensive research on the high yield technology of B. platyphylla seed orchard could be carried out to achieve high and stable yield and improve the economic benefits. At the same time, research on roguing of existing seed orchards and developing of improved seed orchards could improve the genetic quality of seed.

(5) Controlled pollination of $B$. platyphylla could be actively pursued to create excellent combination and selection opportunities, providing selection groups for multi-generation improvement. The foundation for true breeding by artificial mutation is achieved through the control of hybridization, which helps in the understanding of genetic relationships and kinship. The selection and hybridization breeding could be take full advantage for genetic improvement.

(6) Genomic data for B. platyphylla could be intensively studied to facilitate development of molecular markers, genetic maps, gene cloning and functional identification. The transgenic studies might be strengthened to establish an efficient transgenic technology platform and cultivate new lines of transgenic $B$. platyphylla. The metabolic regulatory pathways in $B$. platyphylla conrolling growth, development, wood formation, and stress resistance could be explored, and molecular regulation networks constructed.

Acknowledgements This work was supported by the National High Technology Research and Development Program of China (2013AA102700) and National Key Technology Research and Development Program of the Ministry of Science and Technology of China (2012BAD21B02).

Compliance with ethics guidelines Chuanping Yang declares that he has no conflict of interest or financial conflict to disclose.

This article is a review and does not contain any studies with human or animal subjects performed by the author.

\section{References}

1. Frankel O H, Brown A H D. Current plant genetic resources-a critical appraisa. Genetics: new frontiers (Vol IV). New Delhi: Oxford and IBH Publishing Company, 1984, 1-11

2. Gepts P. Plant genetic resources conservation and utilization: the accomplishments and future of asocietal insurance policy. Crop Science, 2006, 46(5): 2278-2292

3. Wei Z G, Gao Y C, Liu G F, Liu G J, Yang C P. Preliminary construction of core collection of Betula platyphylla germplasm. Scientia Silvae Sinicae, 2009, 45(10): 74-80 (in Chinese)

4. Liu Y, Xu H W, Shang F Q, Jiao H, Zhang L M, Luo J X, Teng W H, Jiang J. Growth stability of Betula platyphylla provenances from three sites. Journal of Beijing Forestry University, 2016, (5): 50-57 (in Chinese)

5. Liu G F, Jiang X B, Liu J C, Li J Y, Wang Y C, Hu R T, Wang H R, Teng W H, Peng H M, Yang C P. Study on the joint analysis of provenances of Betula Platyphylla. Journal of Northeast Forestry University, 1999, (5): 9-14 (in Chinese)

6. Gao F L, Jiang T B. Construction of genetic linkage maps of silver birch based on AFLP markers. Heredita s, 2009,31(2): 213-218 (in Chinese)

7. Jiang J, Yang C P, Liu G F, You X L, Wang Y C. Provence trial of Betula platyphylla Suk. in seedling stage. Journal of Northeast Forestry University, 1999, (6): 1-3 (in Chinese)

8. Zhu X, Liu G F, Yang C P, Liu Z X, Yuan G H, Liu J C, Li J Y. Provenance division and optimal provenance selection of Betula platyphylla. Journal of Northeast Forestry University, 2001, (5): 11-14 (in Chinese)

9. Yang C P, Liu G F, Wei Z G, Wu Y L, Zhou Y M. Study on intensive breeding technique of accelerating Betula platyphyll a flowering and seeding early. Scientia Silvae Sinicae, 2004, 40(6): 75-78

10. Liu X M, Yang C P. Temporal characteristics of developmental cycles of female and male flowers in Betula platyphylla in northeastern China. Scientia Silvae Sinicae,2006, (12): 28-32+ 175 (in Chinese)

11. Wu Y L, Yang C P, Wang Q Y, Zhou Y M, Liu Y.Study on relationship between changes of endogenous hormones and flowering of Betula platyphylla. Journal of Liaoning Forestry Science \& Technology, 2005, (4): 7-8 +47 (in Chinese)

12. Jiang J, Yang C P, Liu G F, Li T H. Anatomic observation on male and female flower bud of Betula platyphylla. Journal of Northeast Forestry University. 2001, (6): 7-10 (in Chinese)

13. Song X S, Li F B, Song F N, Li K L, Liu X M. Morphological correlation of female flower development in birch. Journal of Northeast Forestry University, 2009, (10): 74-80 (in Chinese)

14. Li K L, Jiang J, Jiang Y, Xia D A, Yang C P, Liu G F. Analysis of the genetic effects of seed and seedling traits of Betula platyphylla in a $5 \times 5$ complete diallel cross design. Journal of Northeast Forestry University, 2006, (4): 82-87 (in Chinese)

15. Wang C, Xia D A, Yang C P, Jiang J, Li H Y. The Relation between Hybrid andarents of Betula platyphylla. Journal of Northeast Forestry University, 2004, (02): 1-4 (in Chinese)

16. Wang C, Teng W H, Li K L, Zhang H G, Xia D A, Jiang J. Analysis of genetic effects on growth traits in a $5 \times 5$ diallel cross of Betula platyphylla. Journal of Northeast Forestry University, 2011, (3): 14-20 (in Chinese)

17. Lin L, Mu H Z, Yao Q C, Liu G F, Li H Y, Jiang J. Growth traits of triploid progenies in one year old from different crosses of Betula platyphylla Suk. Journal of Northeast Forestry University, 2012, (5): 1-5 (in Chinese)

18. Liu Y, Xu H W, Li Z X, Jiang J, Peng R S, Shang F Q, Zou J J, Teng 
W H, Liu G F. Growth performance and adaptability of Betula platyphylia offspring in the period of young forest. Journal of Zhejiang $A$ \& F University, 2015, (6): 853-860 (in Chinese)

19. Liu Y, Xu H W, Teng W H, Jiang J, Liu G F. Full-sib progeny test and early selection in superior families of Betula platyphylla. Journal of Northeast Forestry University, 2017, (2): 1-8 (in Chinese)

20. Lin Y, Zhan Y G, Liu Y X, Luan S H, Zhao Z X, Li J Y, Li J T, Ding $\mathrm{Y}$, Lin D B. Anatomical observation on adventitious root formation in softwood cutting of Betula platyphylla. Journal of Northeast Forestry University, 1996, (3): 15-19 (in Chinese)

21. Zhan Y G, Yang C P, Jin Z F, Wang Y C. Endogenous hormones and nutritive material in softwood cuttings of Betula platyphylla during rooting. Journal of Northeast Forestry University, 2001, (4): 1-4 (in Chinese)

22. Zhan Y G, Li J Y, Liu J C, Li L, Teng W H, Wang H R, Qin S H, Liu Y X. Study on propagation techniques of softwood cuttings of Betula platyphylla (I). Journal of Northeast Forestry University, 1994, (2): 6-10 (in Chinese)

23. Li J Y, Zhan Y G, Liu H, Teng W H, Wang H R, Lin Y, Zhang W, Zhang P, Peng H M. Study on propagation techniques of softwood cuttings of Betula platyphylla (II). Journal of Northeast Forestry University, 1995, (3): 10-15 (in Chinese)

24. Su X F, Lin D B, Li L, Xia D A, Jiang Y S, Liu Y X, Yang C P. Relationship between different grafting methods and survival rate of Betula platyphylla. Journal of Northeast Forestry University, 1993, (6): 6-12 (in Chinese)

25. Qiu S Z, Gong Z Q, Liu H N, Zhang D Y, Yang L. Survival influence factors of cutting and grafting for ornamental royal frost Betula. Forest Engineering, 2016, (4): 7-12 + 36 (in Chinese)

26. Tao J, Zhan Y G, You X L, Yang C P, Liu Y X. Study on tissue culture and regeneration system of Betula platyphylla Suks (III) change of endogenous hormonesin tissue culture. Journal of Northeast Forestry University, 1998, (6): 7-10 (in Chinese)

27. Tao J, Zhan Y G, Jiang J, Yang C P, Liu Y X. Study on Tissue Culture and Regenerative System of Betula platyphylla Suks (I). Journal of Northeast Forestry University, 1998, (5): 7-10 (in Chinese)

28. Tao J, Zhan Y G, Jiang J, Yang C P, Liu Y X. Study on Tissue Culture and Regenerative System of Betula platyphylla Suks (II). Journal of Northeast Forestry University, 1998, (6): 1-5 (in Chinese)

29. Liu F M, Mu H Z, Liu Z J, Li Z X, Jiang J, Liu G F. Inducing tetraploid of Betula platyphylla with different generations of seeds by colchicine. Journal of Beijing Forestry University, 2013, (3): 84 89 (in Chinese)

30. Mu H Z, Liu Z J, Lin L, Li H Y, Jiang J, Liu G F. Transcriptomic analysis of phenotypic changes in birch (Betula platyphylla) autotetraploids. International Journal of Molecular Sciences, 2012, 13(10): 13012-13029

31. Wang S, Zhao H, Li M Y, Jiang J.Analysis of IAA and ABA in leaf buds of different ploidy Betula platyphyllaSuk. Journal of Anhui Agricultural Sciences,2014, (27): 9418-9420 (in Chinese)

32. Du L, Li Y C, Mu H Z, Zhang T Y, Liu F F, Huang H J, Liu G F. Photosynthetic characteristics of tetraploid and diploid Betula platyphylla. Journal of Northeast Forestry University, 2011, (2):
1-4 (in Chinese)

33. Mu HZ, Lin L, Yu JG, Jiang X L, Zhang X, Xia F C. Morphology and hysiology of autotetraploid Betula platyphylla responding to $\mathrm{NaHCO}_{3}$ stress. Journal of Beihua University (Natural Science), 2016, (5): 585-589 (in Chinese)

34. Wang S, Zhao H, Yang C P, Jiang J. Analysis and evaluation of triterpenoids in tetraploid birch (Betula). Journal of Northeast Forestry University, 2015, (9): 53-61 (in Chinese)

35. Xu H W, Liu Y, Li Y J, Teng W H, Jiang J, Zhao X Y. Comparative analysis on seed vitality of different families produced in Betula pendula triploid seed orchard. Journal of Southwest Forestry College, 2013, (5): 34-39 (in Chinese)

36. Liu Y, Xu H W, Li Z X, Teng W H, Zhang L F, Zou J J, Shang F Q, Liu G F. Growth variation and stability analysis of birch crossbreed families. Bulletin of Botanical Research, 2015, (6): 937-944 (in Chinese)

37. Jiang J, Jiang Y, Yang CP, Guo M, Li K L, Liu G F, Li H Y. A preliminary study on space mutation breeding in Betula platyphylla. Journal of Nuclear Agricultural Sciences, 2006, (1): 27-31 (in Chinese)

38. Huang H J, Li K L, Liu G F, Li Z X, Teng W H, Jiang J. Preliminary study on early growth traits of Betula platyphylla. Journal of Nuclear Agricultural Sciences, 2010, (6): 1148-1151 (in Chinese)

39. Mu H, Lin L, Liu G, Jiang J. Transcriptomic analysis of incised leafshape determination in birch. Gene, 2013, 531(2): 263-269

40. Lin L, Mu H, Jiang J, Liu G. Transcriptomic analysis of purple leaf determination in birch. Gene, 2013, 526(2): 251-258

41. Qu G Z, Zheng T, Liu G, Wang W, Zang L, Liu H, Yang C. Overexpression of a MADS-box gene from birch (Betula platyphylla) promotes flowering and enhances chloroplast development in transgenic tobacco. PLoS One, 2013, 8(5): e63398

42. Xing L, Liu X M. Characterization of Betula platyphylla gene transcripts associated with early development of male inflorescence. Molecular Biology Reports, 2012, 39(2): 929-935

43. Yin J, Ren C L, Zhan Y G, Li C X, Xiao J L, Qiu W, Li X Y, Peng H M. Distribution and expression characteristics of triterpenoids and OSC genes in white birch (Betula platyphylla suk.). Molecular Biology Reports, 2012, 39(3): 2321-2328

44. Zhang R, Yang C, Wang C, Wei Z, Xia D, Wang Y, Liu G, Wang Y. Time-course analyses of abscisic acid level and the expression of genes involved in abscisic acid biosynthesis in the leaves of Betula platyphylla. Molecular Biology Reports, 2012, 39(3): 2505-2513

45. Liu X, Wang Q, Chen P, Song F, Guan M, Jin L, Wang Y, Yang C. Four novel cellulose synthase (CESA) genes from Birch (Betula platyphylla Suk.) involved in primary and secondary cell wall biosynthesis. International Journal of Molecular Sciences, 2012, 13 (10): 12195-12212

46. Liu X, Liu Y, Liu C, Guan M, Yang C. Identification of genes associated with male sterility in a mutant of white birch (Betula platyphylla Suk.). Gene, 2015, 574(2): 247-254

47. Zhang W, Yang G, Mu D, Li H, Zang D, Xu H, Zou X, Wang Y. An ethylene-responsive factor BpERF11 negatively modulates salt and osmotic tolerance in Betula platyphylla. Scientific Reports, 2016, 6 (1): 23085

48. Zhan Y G, Wang Y C, Wang Z Y, Yang C P, Liu Z H, Li C H. 
Genetic transformation of Betula platyphylla and insect resistance of transgenic plants. Journal of Plant Physiology and molecular biology, 2003, (5): 380-386 (in Chinese)

49. Zeng F, Qian J, Luo W, Zhan Y, Xin Y, Yang C. Stability of transgenes in long-term micropropagation of plants of transgenic birch (Betula platyphylla). Biotechnology Letters, 2010, 32(1): 151156

50. Zhang Y, Wang Y, Wang C. Gene overexpression and gene silencing in birch using an Agrobacterium-mediated transient expression system. Molecular Biology Reports, 2012, 39(5): $5537-5541$

51. Wang Z Y, Fan H J, Xue Z, Zhan Y. Division of resistance grades of transgenic birch and its influence on larval midgut. 2005, (3): 38-39 +66 (in Chinese)

52. Zhang W, Wei R, Chen S, Jiang J, Li H, Huang H, Yang G, Wang S, Wei H, Liu G. Functional characterization of CCR in birch (Betula platyphylla $\times$ Betula pendula) through overexpression and suppression analysis. Physiologia Plantarum, 2015, 154(2): 283296

53. Huang H, Chen S, Li H, Jiang J. Next-generation transcriptome analysis in transgenic birch overexpressing and suppressing APETALA1 sheds lights in reproduction development and diterpenoid biosynthesis. Plant Cell Reports, 2015, 34(9): 1663-1680

54. Guo H, Wang Y, Wang L, Hu P, Wang Y, Jia Y, Zhang C, Zhang Y, Zhang Y, Wang C, Yang C. Expression of the MYB transcription factor gene BplMYB46 affects abiotic stress tolerance and secondary cell wall deposition in Betula platyphylla. Plant Biotechnology Journal, 2017, 15(1): 107-121

55. Zhao X, Zheng T, Shao L, Xiao Z, Wang F, Li S, Zang L, Zheng M, Li Y, Qu G Z. Variation analysis of physiological traits in Betula platyphylla overexpressing TaLEA-ThbZIP gene under salt stress. PLoS One, 2016, 11(11): e0164820

56. Zhou C, Li C. A novel R2R3-MYB transcription factor BpMYB106 of birch (Betula platyphylla) confers increased photosynthesis and growth rate through up-regulating photosynthetic gene expression. Frontiers in Plant Science, 2016, 7: 315

57. Wang C, Wang Y C, Diao G P, Jiang J, Yang C P. Isolation and characterization of expressed sequence tags (ESTs) from cambium tissue of birch (B. platyphylla). Plant Molecular Biology Reporter, 2010, 28(3): 438-449
58. Guo H Y, Wang Y C, Hu P, Wang Y M, Jiang Y, Yang C P, Wang C. Gene expression profiles in different stem internodes reveal the genetic regulation of primary and secondary stem development in Betula platyphylla. Tree Genetics \& Genomes, 2016, 12(6): 113

59. Guo H, Wang Y, Liu H, Hu P, Jia Y, Zhang C, Wang Y, Gu S, Yang $\mathrm{C}$, Wang C. Exogenous $\mathrm{GA}_{3}$ application enhances xylem development and induces the expression of secondary wall biosynthesis related genes in Betula platyphylla. International Journal of Molecular Sciences, 2015, 16(9): 22960-22975

60. Jiang T B, Li S C, Gao F L, Ding B J, Qu Y J, Tang X H, Liu G F, Jiang J, Yang C P. Genetic linkage map of Betula pendula Roth. and Betula platyphylla Suk. based on random amplified polymorphisms DNA markers. Hereditas, 2007, (7): 867-873 (in Chinese)

61. Wang Y M, Wei Z G, Yang C P. Dateming for SSRs in ESTs and EST-SSR marker development in Betula platyphylla. Scientia Silvae Sinicae, 2008, (9): 1-4 (in Chinese)

62. Wang D, Wei Z G, Yang C P, Liu G J. Analysis and identification of SCAR molecular markers associated with birch fiber length trait. Journal of Forestry Research, 2008, (4): 288-292+350 (in Chinese)

63. Zhang X L, Zhang K X, Yang C P, Qu G Z, Liu G F, Wei Z G. Construction and optinization of SPAR rection system for Betula platyphylla. Journal of Northeast Forestry University, 2010, (9): 13 (in Chinese)

64. Wei Z G, Yang C P, Pan H. Identification of molecular markers associated with birch fiber length trait by multiple regression analysis. Molecular Plant Breeding, 2006, (6): 835-840

65. Xia D, Wei Z G, Yang C P, Liu G J. Analysis of ISSR and SCAR marker associated with birch fiber length trait. Journal of Northeast Forestry University, 2008, (9): 1-4 (in Chinese)

66. Lv C Y, Zhou B R, Wang L, Wu L L, Jiang T B. Genetic linkage maps of Betula platyphylla Suk. and Betula pendula Roth. based on RAPD and AFLP. Plant Physiology, 2009, (8): 775-780

67. Gao Y C, Wei Z G, Yang C P, Liu G F, Liu G J. A 10-year-old provenance trial of Betula platyphylla in the Maoershanarea of Heilongiiang Province. Journal of Zhejiang Forestry College, 2016, (5): 50-57 (in Chinese)

68. Li S C, Gao F L, Jiang T B. Analysis of genetic linkage groups on birch using RAPD markers. Scientia Silvae Sinicae, 2008, (5): 155159 (in Chinese) 